\title{
Planar Central Configurations of Symmetric Five-Body Problems with Two Pairs of Equal Masses
}

\author{
Muhammad Shoaib, ${ }^{1,2}$ Abdul Rehman Kashif, ${ }^{1}$ and Anoop Sivasankaran ${ }^{3}$ \\ ${ }^{1}$ Department of Mathematics, University of Ha'il, P.O. Box 2440, Ha'il 81451, Saudi Arabia \\ ${ }^{2}$ Abu Dhabi Men's College, Higher Colleges of Technology, P.O. Box 25035, Abu Dhabi, UAE \\ ${ }^{3}$ Applied Mathematics and Sciences, Khalifa University of Science Technology and Research, P.O. Box 127788, Abu Dhabi, UAE
}

Correspondence should be addressed to Anoop Sivasankaran; anoop.sivasankaran@kustar.ac.ae

Received 2 November 2015; Revised 11 January 2016; Accepted 18 January 2016

Academic Editor: Elmetwally Elabbasy

Copyright ( $) 2016$ Muhammad Shoaib et al. This is an open access article distributed under the Creative Commons Attribution License, which permits unrestricted use, distribution, and reproduction in any medium, provided the original work is properly cited.

We study central configuration of a set of symmetric planar five-body problems where (1) the five masses are arranged in such a way that $m_{1}, m_{2}$, and $m_{4}$ are collinear and $m_{2}, m_{3}$, and $m_{5}$ are collinear; the two sets of collinear masses form a triangle with $m_{2}$ at the intersection of the two sets of collinear masses; (2) four of the bodies are on the vertices of an isosceles trapezoid and the fifth body can take various positions on the axis of symmetry both outside and inside the trapezoid. We form expressions for mass ratios and identify regions in the phase space where it is possible to choose positive masses which will make the configuration central. We also show that the triangular configuration is not possible.

\section{Introduction}

The equations of motion for $n$ positive masses subject to Newtonian Gravitation is given by

$$
m_{i} \ddot{\overrightarrow{r_{i}}}=\nabla_{i} U_{i}, \quad i=1, \ldots, n,
$$

where

$$
U=-G \sum_{i=1}^{N} \sum_{j=1}^{j<i} \frac{m_{i} m_{j}}{\left|r_{i}-r_{j}\right|}
$$

is the Newtonian potential, $r_{i}$ is the position vector of the $i$ th body, $m_{i}$ is the mass of the $i$ th body, and $G$ is the universal constant of gravitation. The central configuration of an $n$ body system is obtained, if the position vector of each particle with respect to the center of mass is a common scalar multiple of its acceleration; that is,

$$
\ddot{\overrightarrow{r_{i}}}=\lambda r_{i}, \quad i=1, \ldots, n,
$$

where the constant $\lambda \neq 0$.
Using the Laura/Andoyer/Dziobek equations [1], the condition that $\left(m_{1}, m_{2}, \ldots, m_{n}\right)$ form a planar, noncollinear, central configuration is

$$
f_{i j}=\sum_{k=1, k \neq i, j}^{n} m_{k}\left(R_{i k}-R_{j k}\right) \Delta_{i j k}=0,
$$

where $R_{i j}=1 / r_{i j}^{3}$ and $\Delta_{i j k}=\left(r_{i}-r_{j}\right) \wedge\left(r_{i}-r_{k}\right)$.

The study of the central configurations plays a key role in understanding the Gravitational $n$-body problems (cf. [2-4]). Central configurations are useful in providing explicit homographic solutions of the equations of motion and families of periodic solutions [5]. They are also useful in understanding the nature of solutions near collisions and the energy level sets that hold the central configuration determine the topology of the integral manifolds. Understanding the four- and five-body problem is very important as it is known that approximately two-thirds of stars in our Galaxy are part of multistellar systems. There is a growing interest in studying spatial central configurations of Newtonian five-body problem with various symmetrical restrictions. Several authors 
have recently studied central configuration of restricted fourbody and five-body problems [6-9]. According to [10], finiteness problem of $n$-body central configurations is an open problem for the 21st century. Hampton and Moeckel [11] proved that finite central configurations are possible for the Newtonian three-body and four-body problems with positive masses. Finiteness of central configurations of planar fivebody problem is recently established by Albouy and Kaloshin in [12]. Hampton and Jensen [13] showed that number of spatial central configurations are finite for positive masses in the Newtonian five-body problem.

Several authors studied the inverse problem of central configurations by finding the mass vectors which makes the configuration central for a given configuration of $n$ bodies. Albouy and Moeckel [14] show that two-parameter family of masses exists for collinear central configurations with $n \leq 6$. Xie [15] proved the existence of a singular curve where it is possible to have permutational admissible set of mass vectors in the collinear central configuration fourbody problem. According to [16], families of stacked central configurations can be formed by adding extra bodies to known central configurations of three bodies. Gidea and Llibre [1] studied the stacked symmetric planar central configuration of five bodies with some special symmetries. They have shown that central configuration is possible in rhomboidal arrangement where four masses are kept at the vertices and a fifth mass in the center and a trapezoidal arrangement where four masses are at the vertices and a fifth mass at the midpoint of one of the parallel sides. Shoaib et al. [17] have studied central configuration of the rhomboidal 5-body problem and identified CC regions using similar approaches.

Xie [18] studied central configuration of the planar Newtonian four-body problems, where two equal mass pairs are kept at adjacent vertices of a trapezoid and possible central configuration mass ratios are expressed in terms of the size of quadrilateral. While much is known about trapezoid central configurations in the restricted four-body problem, there is less known about trapezoid central configurations in the restricted five-body problem. Shoaib [19] recently investigated the inverse problem of central configuration in a symmetric $4+1$-body problem and derived regions of central configuration.

In this work, we study the central configuration of the isosceles trapezoidal five-body problem and identify regions in the phase space where it is possible to choose positive masses which will make the configuration central. We are motivated by the work of [1] and follow similar ideas to study planar symmetric five-body problems. The problems we investigate include two types of trapezoidal five-body problems with four masses on its vertices and a fifth mass on the axis of symmetry which can be both inside and outside the trapezoid. The third type of problem which is investigated here is a triangular problem with two pairs of masses and a fifth mass on the perpendicular bisector of the triangle which is not necessarily equal to any of the four masses. In Section 2, we present the main theorems related to the three five-body problems and their proofs are given in Sections 3, 4, and 5, respectively.

\section{Main Results}

Theorem 1. Consider a 5-body noncollinear configuration $\vec{r}=\left(\mathbf{r}_{1}, \mathbf{r}_{2}, \mathbf{r}_{3}, \mathbf{r}_{4}, \mathbf{r}_{5}\right)$ and a positive mass vector $\left(m_{1}, m_{2}, m_{3}\right.$, $\left.m_{4}, m_{5}\right)$, where

$$
\begin{aligned}
& \mathbf{r}_{1}=(-1,0), \\
& \mathbf{r}_{2}=(0,-w), \\
& \mathbf{r}_{3}=(1,0), \\
& \mathbf{r}_{4}=(-s, t), \\
& \mathbf{r}_{5}=(s, t) .
\end{aligned}
$$

Assume that $m_{1}=m_{3}$ and $m_{4}=m_{5}$ are placed symmetrically on the vertices of an isosceles trapezoid and the fifth mass is placed on the axis of symmetry of the trapezoid.

(a) When $s=t$, there is a continuous family of central configurations determined by the region $R$ and the function $C(t, w)=0$, given in Figure 2. There are no central configurations when $w>0$.

(b) When $t=1$ and $s \in(0,0.64)$ there exists a continuous family of central configurations for $w>0$. There are no central configurations when $w<0$ or when $s>0.64$.

Theorem 2. Consider a 5-body noncollinear configuration $\vec{r}=\left(\mathbf{r}_{1}, \mathbf{r}_{2}, \mathbf{r}_{3}, \mathbf{r}_{4}, \mathbf{r}_{5}\right)$ with a positive mass vector $\left(m_{1}, m_{2}, m_{3}\right.$, $\left.m_{4}, m_{5}\right)$, where $\mathbf{r}_{i}, i=1,2, \ldots, 5$ are given by (5). The five masses are arranged in such a way that $m_{1}, m_{2}$, and $m_{4}$ are collinear and $m_{2}, m_{3}$, and $m_{5}$ are collinear. The two sets of collinear masses form a triangle with $m_{2}$ at the intersection of the two sets of collinear masses. Then there does not exist any such type of central configuration.

\section{Proof of Theorem 1}

3.1. Proof of Theorem 1(a). Consider five bodies of masses $m_{1}$, $m_{2}, m_{3}, m_{4}$, and $m_{5}$ placed at $\mathbf{r}_{1}, \mathbf{r}_{2}, \mathbf{r}_{3}, \mathbf{r}_{4}$, and $\mathbf{r}_{5}$ :

$$
\begin{aligned}
& \mathbf{r}_{1}=(-1,0), \\
& \mathbf{r}_{2}=(0,-w), \\
& \mathbf{r}_{3}=(1,0), \\
& \mathbf{r}_{4}=(-t, t), \\
& \mathbf{r}_{5}=(t, t) .
\end{aligned}
$$

Four of the masses make a trapezoid (see Figure 1) while the mass $m_{2}$ is on the line of symmetry. Using the inherent symmetries of the trapezoidal 5-body model we obtain the following system of four equations from (4) which define the central configurations for the model described above: 


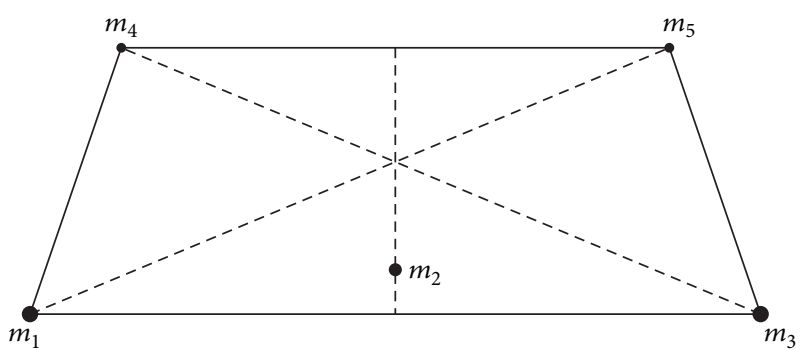

Figure 1: Trapezoidal five-body configuration.

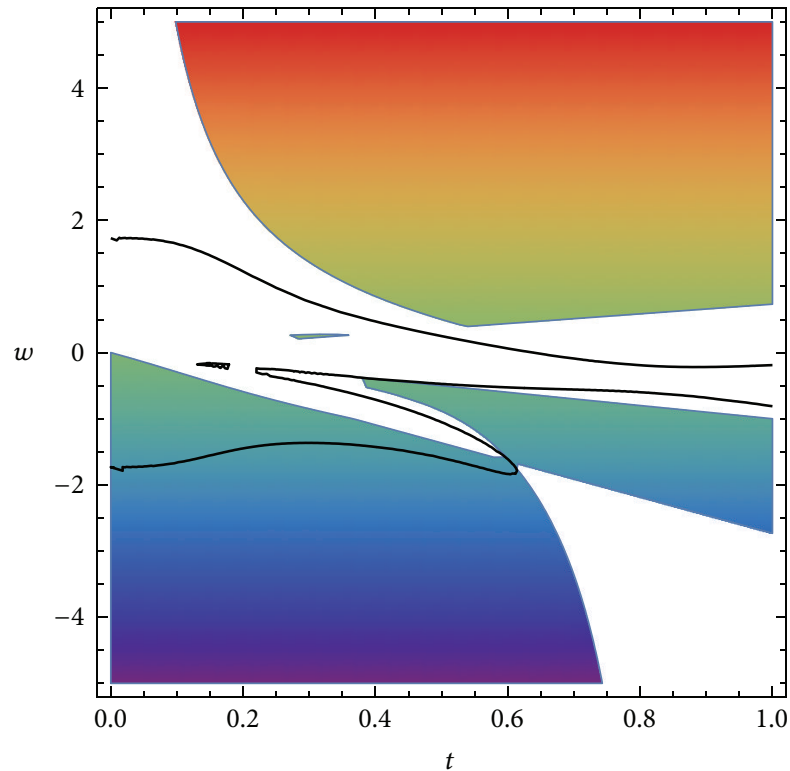

FIGURE 2: Central configuration region $R=R_{\mu_{1}} \cap R_{\mu_{2}}$ (shaded), where both $\mu_{1}$ and $\mu_{2}$ are positive. The bold line corresponds to $C(t, w)=0$.

$$
\begin{aligned}
& f_{12}=m_{1} h_{31}+m_{4} h_{33}=0, \\
& f_{14}=m_{1} h_{11}+m_{2} h_{12}+m_{4} h_{13}=0, \\
& f_{15}=m_{1} h_{21}+m_{2} h_{22}+m_{4} h_{23}=0, \\
& f_{24}=m_{1} h_{41}+m_{4} h_{43}=0,
\end{aligned}
$$

where

$$
\begin{aligned}
& h_{11}=\left(R_{13}-R_{15}\right) \Delta_{143}, \\
& h_{12}=\left(R_{24}-R_{12}\right) \Delta_{124}, \\
& h_{13}=\left(R_{15}-R_{45}\right) \Delta_{145}, \\
& h_{21}=\left(R_{13}-R_{14}\right) \Delta_{143}, \\
& h_{22}=\left(R_{24}-R_{12}\right) \Delta_{125}, \\
& h_{23}=\left(R_{45}-R_{14}\right) \Delta_{145}, \\
& h_{31}=\left(R_{13}-R_{12}\right) \Delta_{123},
\end{aligned}
$$

$$
\begin{aligned}
h_{33} & =\left(R_{14}-R_{24}\right) \Delta_{124}+\left(R_{15}-R_{24}\right) \Delta_{125}, \\
h_{41} & =\left(R_{12}-R_{14}\right) \Delta_{124}+\left(R_{23}-R_{34}\right) \Delta_{243}, \\
h_{43} & =\left(R_{24}-R_{45}\right) \Delta_{245}, \\
R_{12} & =R_{23}=\left(1+w^{2}\right)^{-3 / 2}, \\
8 R_{13} & =1,
\end{aligned}
$$$$
R_{14}=R_{35}=\left((1-t)^{2}+t^{2}\right)^{-3 / 2},
$$$$
R_{15}=R_{34}=\left((1+t)^{2}+t^{2}\right)^{-3 / 2},
$$$$
R_{24}=R_{25}=\left((t+w)^{2}+s^{2}\right)^{-3 / 2},
$$$$
8 R_{45}=t^{-3} \text {, }
$$$$
\Delta_{124}=\Delta_{235}=(t+w(1-s)),
$$$$
\Delta_{125}=\Delta_{234}=(t+w(1+t)) \text {, }
$$$$
\Delta_{145}=\Delta_{345}=-2 t^{2},
$$$$
\Delta_{245}=-2 t(t+w) \text {, }
$$$$
\Delta_{123}=2 w \text {. }
$$

Write (7) to (10) as a linear homogeneous system in $\left(m_{1}, m_{2}, m_{4}\right)$ given by the matrix

$$
M=\left(\begin{array}{ccc}
h_{41} & 0 & h_{43} \\
h_{31} & 0 & h_{33} \\
h_{21} & h_{22} & h_{23} \\
h_{11} & h_{12} & h_{13}
\end{array}\right) .
$$

Using a simple Gaussian elimination approach, matrix $M$ is reduced as in what follows:

$$
\left(\begin{array}{ccc}
h_{41} & 0 & h_{43} \\
0 & h_{22} & -\frac{1}{h_{41}}\left(h_{21} h_{43}-h_{23} h_{41}\right) \\
0 & 0 & -\frac{1}{h_{41}}\left(h_{31} h_{43}-h_{41} h_{33}\right) \\
0 & 0 & 0
\end{array}\right)
$$

The above systems can now be written in equation form as in what follows:

$$
\begin{aligned}
m_{1} h_{41}+m_{4} h_{43} & =0 \\
m_{2} h_{22}-\frac{m_{4}}{h_{41}}\left(h_{21} h_{43}-h_{23} h_{41}\right) & =0 \\
m_{4}\left(h_{31} h_{43}-h_{41} h_{33}\right) & =0
\end{aligned}
$$

For the above systems to have a nontrivial solution $h_{31} h_{43}-$ $h_{41} h_{33}=0$. This will be treated as a geometric constraint 
on the existence of trapezoidal central configurations. Setting $\mu_{1}=m_{1} / m_{4}$ and $\mu_{2}=m_{2} / m_{4}$, we obtain

$$
\begin{aligned}
& \mu_{1}(t, w)=-\frac{h_{43}}{h_{41}} ; \\
& \mu_{2}(t, w)=\frac{h_{21} h_{43}-h_{23} h_{41}}{h_{41} h_{22}}=\frac{N_{\mu_{2}}(t, w)}{D_{\mu_{2}}(t, w)} .
\end{aligned}
$$

Such that $h_{21} \neq 0$ and $h_{41} \neq 0$. Therefore (15) define central configuration for the trapezoidal 5-body problem for all masses subject to the constraint

$$
C(t, w)=h_{31} h_{43}-h_{41} h_{33}=0 .
$$

It can be seen from the reduced matrix that (8) is not used in deriving $\mu_{1}$ and $\mu_{2}$ which necessitates a second constraint given as follows:

$$
\begin{aligned}
C^{*}(t, w)= & h_{43}\left(h_{12} h_{21}-h_{11} h_{22}\right) \\
& +h_{41}\left(h_{13} h_{22}-h_{12} h_{23}\right)=0 .
\end{aligned}
$$

However it is numerically confirmed that $C^{*}(t, w)=0$ is satisfied everywhere, where $C(t, w)=0$. The constraint $C^{*}(t, w)=0$ has some additional solutions but that is irrelevant as for nontrivial solution both the constraints have to be satisfied. Hence we will only use $C(t, w)$ in our analysis. As only positive solutions are of practical interest, therefore we will now isolate the regions where all the masses are positive.

Lemma 3. The function $\mu_{1}(t, w)=-h_{43} / h_{41}$ attain positive values in

$$
R_{\mu_{1}}=\left(R_{h_{43^{-}}} \cap R_{h_{41^{+}}}\right) \cup\left(\left(R_{h_{43^{-}}}\right)^{c} \cap\left(R_{h_{41^{+}}}\right)^{c}\right),
$$

where $R_{h_{43^{-}}}$and $R_{h_{41^{+}}}$are given in (20) and (22).

Proof. To find the central configuration region where $\mu_{1}$ is positive, we will need to find regions in the tw-plane where $h_{43}$ and $h_{41}$ have opposite signs. We can see that

$$
h_{43}=2 t(t+w)\left(\frac{1}{8 t^{3}}-\frac{1}{\left(t^{2}+(t+w)^{2}\right)^{3 / 2}}\right)
$$

is negative when its two factors have opposite signs. Therefore $h_{43}<0$ in

$$
\begin{aligned}
& R_{h_{43^{-}}}=\{(t, w) \mid(w<0 \wedge(t<-0.37 w \vee t>-w)) \\
& \quad \vee(w>0 \wedge t>1.37 w)\} .
\end{aligned}
$$

Similarly

$$
\begin{aligned}
h_{41}= & ((1-t) w+t) \\
& \cdot\left(\left(w^{2}+1\right)^{-3 / 2}-\left(t^{2}+(1-t)^{2}\right)^{-3 / 2}\right) \\
& +((t+1) w+t) \\
& \cdot\left(\left(t^{2}+(t+1)^{2}\right)^{-3 / 2}-\left(w^{2}+1\right)^{-3 / 2}\right) .
\end{aligned}
$$

As $h_{41}$ is a sum of two nonlinear functions of $t$ and $w$, we used some approximation techniques to find the region in the $t w$ plane, where $h_{41}>0$ and

$$
\begin{aligned}
& R_{h_{41^{+}}} \\
& \quad=\left\{(t, w) \mid\left(w<0 \wedge t>0.5 \sqrt{2 w^{2}+3.5}+0.1\right)\right\} .
\end{aligned}
$$

Thus the CC region where $\mu_{1}$ has positive real values is given as follows:

$$
R_{\mu_{1}}=\left(R_{h_{43^{-}}} \cap R_{h_{41^{+}}}\right) \cup\left(\left(R_{h_{43^{-}}}\right)^{c} \cap\left(R_{h_{41^{+}}}\right)^{c}\right) .
$$

This completes the proof of Lemma 3.

Lemma 4. The function $D_{\mu_{2}}(t, w)=h_{41} h_{22}$ attain positive values in the following region of the tw-plane:

$$
R_{D_{\mu_{2}}}=\left(R_{h_{22^{+}}} \cap R_{h_{41^{+}}}\right) \cup\left(\left(R_{h_{22^{+}}}\right)^{c} \cap\left(R_{h_{41^{+}}}\right)^{c}\right) .
$$

Proof. The denominator of $\mu_{2}$, that is, $D_{\mu_{2}}$, is positive when its components $\left(h_{22}, h_{41}\right)$ have the same signs. We have shown that $h_{41}>0$ in $R_{h_{41^{+}}}$. The second component

$$
\begin{aligned}
h_{22}= & ((t+1) w+t) \\
& \cdot\left(\left(t^{2}+(t+w)^{2}\right)^{-3 / 2}-\left(w^{2}+1\right)^{-3 / 2}\right)
\end{aligned}
$$

is positive in

$$
\begin{aligned}
& R_{h_{22^{+}}}=\left\{(t, w) \mid\left(0<t<1 \wedge-t(t+1)^{-1}<w\right.\right. \\
& \left.\left.\quad<\left(1-2 t^{2}\right)(2 t)^{-1}\right)\right\} .
\end{aligned}
$$

Hence $D_{\mu_{2}}>0$ in $R_{D_{\mu_{2}}}=\left(R_{h_{22^{+}}} \cap R_{h_{41^{+}}}\right) \cup\left(\left(R_{h_{22^{+}}}\right)^{c} \cap\left(R_{h_{41^{+}}}\right)^{c}\right)$.

Lemma 5. The function $N_{\mu_{2}}(t, w)=h_{21} h_{43}-h_{23} h_{41}$ attain positive values in the following region of tw-plane:

$$
R_{N_{\mu_{2}}}=R_{N_{\mu_{2}} a} \cup R_{N_{\mu_{2}} b} \cup R_{N_{\mu_{2}} c} \cup R_{N_{\mu_{2}} d}
$$

Proof. Using the same technique as we have used for $h_{22}, h_{41}$, and $h_{43}$ we show that $h_{21}, h_{23}$, and $h_{43}$ are positive in the following regions:

$$
\begin{aligned}
& R_{h_{21^{+}}}=\{(t, w) \mid 0<t<1 \wedge w \in \mathbb{R}\}, \\
& R_{h_{23^{+}}}=\{(t, w)|(t, w)| 0.37<t<1 \wedge w \in \mathbb{R}\}, \\
& R_{h_{43^{+}}}=\left(R_{h_{43^{-}}}\right)^{c} .
\end{aligned}
$$

There are various possibilities for $N_{\mu_{2}}$ to have positive values. These possibilities are listed below with the corresponding regions:

(a) $h_{21}>0, h_{43}>0, h_{23}<0, h_{41}>0$. Consider

$$
\begin{aligned}
& R_{N_{\mu_{2}} a}=R_{h_{21^{+}}} \cap R_{h_{43^{+}}} \cap\left(R_{h_{23^{+}}}\right)^{c} \cap R_{h_{41^{+}}} \\
& \quad=\{(t, w) \mid(-1<w \leq-0.37 \wedge-0.37 w<t<0.37) \\
& \quad \vee(-0.37<w<0 \wedge-0.37 w<t<-w)\} .
\end{aligned}
$$


(b) $h_{21}>0, h_{43}>0, h_{23}>0, h_{41}<0$. Consider

$$
\begin{aligned}
& R_{N_{\mu_{2}} b}=R_{h_{21^{+}}} \cap R_{h_{43^{+}}} \cap R_{h_{23^{+}}} \cap\left(R_{h_{41^{+}}}\right)^{c} \\
& \quad=\{(t, w) \mid(-2.7<w \leq-1 \wedge-0.37 w<t<1) \\
& \quad \vee(-1<w<-0.37 \wedge 0.37<t<-w) \\
& \quad \vee(w>0 \wedge 0.36<t<1)\} .
\end{aligned}
$$

The combination $h_{21}<0, h_{43}<0, h_{23}>0$, $h_{41}<0$ and $h_{21}<0, h_{43}<0, h_{23}<0, h_{41}>0$ returned empty regions. It is also possible for $N_{\mu_{2}}$ to be positive in some part of the region where at least one of $\left(h_{21} h_{43}\right)$ and $\left(-h_{23} h_{41}\right)$ are positive. An all inclusive analytical solution is not possible in most of these cases; therefore we will use approximation techniques to find regions where $N_{\mu_{2}}$ is positive.

(c) $h_{21}>0, h_{43}>0, h_{23}<0, h_{41}<0 . N_{\mu_{2}}>0$ in the following region:

$$
\begin{aligned}
& R_{h_{21^{+}}} \cap R_{h_{43^{+}}} \cap\left(R_{h_{23^{+}}}\right)^{c} \cap\left(R_{h_{41^{+}}}\right)^{c} \\
& \quad=\{(t, w) \mid(0<w<0.27 \wedge 0<t<1.37 w) \\
& \quad \vee(w \geq 0.27 \wedge 0<t<0.37)\}=R_{N_{\mu_{2}} \cdot}
\end{aligned}
$$

In this case $N_{\mu_{2}}$ is never negative.

(d) $h_{21}>0, h_{43}<0, h_{23}>0, h_{41}<0$. This region is determined from $R_{h_{21^{+}}} \cap\left(R_{h_{43^{+}}}\right)^{c} \cap R_{h_{23^{+}}} \cap\left(R_{h_{41^{+}}}\right)^{c}$

$$
\begin{aligned}
R_{N_{\mu_{2}}} d^{*} & =\{(w \leq-5 \wedge 0.37<t<1) \\
& \vee(-5<w \leq-1.8 \wedge 0.72<t<-0.37 w) \\
& \vee(-1<w \leq-0.37 \wedge-w<t<1) \\
& \vee(-0.37<w \leq 0.27 \wedge 0.37<t<1) \\
& \vee(0.27<w<1.3 \wedge 1.37 w<t<1)\} .
\end{aligned}
$$

Using numerical approximation techniques it can be shown that $N_{\mu_{2}}>0$ in the following part of $R_{N_{\mu_{2}} d^{*}}$ :

$$
\begin{aligned}
& R_{N_{\mu_{2}} d} \\
& \quad=\{(t, w) \mid(-5<w \leq-1.8 \wedge 0.8<t<-0.37 w) \\
& \quad \vee(-1<w \leq-0.7 \wedge-w<t<1) \\
& \quad \vee(0.1<w<1.3 \wedge 1.37 w<t<1)\} .
\end{aligned}
$$

$R_{N_{\mu_{2}}}$ is always negative in the rest of the combinations of $h_{21}$, $h_{43}, h_{23}$, and $h_{41}$. Therefore $N_{\mu_{2}}>0$ in the union of the regions found above. This completes the proof of Lemma 5.

Lemma 6. Given the five-body arrangement of Theorem $1, R_{\mu_{2}}$ defines the region of central configurations where $\mu_{2}>0$.
Proof. For $\mu_{2}>0, N_{\mu_{2}}$ and $D_{\mu_{2}}$ must have the same sign. Therefore central configuration region where $\mu_{2}>0$ is given by

$$
R_{\mu_{2}}=\left(R_{N_{\mu_{2}}} \cap R_{D_{\mu_{2}}}\right) \cup\left(\left(R_{N_{\mu_{2}}}\right)^{c} \cap\left(R_{D_{\mu_{2}}}\right)^{c}\right) .
$$

This completes the proof of Lemma 6 .

The proof of Theorem 1(a) is a direct consequence of Lemmas 3 and 6 . That is, the central configuration region $R=R_{\mu_{1}} \cap R_{\mu_{2}}$, where both $\mu_{1}$ and $\mu_{2}$ are positive, can be found by taking the intersection of the regions found for $\mu_{1}$ and $\mu_{2}$. This region is given in Figure 2 with the geometric constraint $C(t, w)=0$. The continuous family of central configurations is shown by intersection of the bold line with the colored region in Figure 2. This completes the proof of Theorem 1.

3.2. Proof of Theorem 1(b). Consider five bodies of masses $m_{1}$, $m_{2}, m_{3}, m_{4}$, and $m_{5}$ placed at $\mathbf{r}_{1}=(-1,0), \mathbf{r}_{2}=(0,-w), \mathbf{r}_{3}=$ $(1,0), \mathbf{r}_{4}=(-s, 1)$, and $\mathbf{r}_{5}=(s, 1)$, respectively. Similar to Theorem 1, four of the masses make a trapezoid (see Figure 1) while the mass $m_{2}$ is on the line of symmetry. In Theorem 1, the trapezoid considered had $s=t$. In this case, the height of trapezoid is fixed at 1 with variable width. The general CC equations are the same as in Theorem 1 with different $R_{i j}$ 's. The $R_{i j}$ which have different values are

$$
\begin{aligned}
R_{12} & =R_{23}=\left(1+w^{2}\right)^{-3 / 2}, \\
8 R_{13} & =1, \\
R_{14} & =R_{35}=\left((1-s)^{2}+1\right)^{-3 / 2}, \\
R_{15} & =R_{34}=\left((1+s)^{2}+1\right)^{-3 / 2}, \\
R_{24} & =R_{25}=\left((1+w)^{2}+s^{2}\right)^{-3 / 2}, \\
8 R_{45} & =s^{-3}, \\
\Delta_{124} & =\Delta_{235}=(1+w(1-s)), \\
\Delta_{125} & =\Delta_{234}=(1+w(1+s)), \\
\Delta_{145} & =\Delta_{345}=-2 s, \\
\Delta_{245} & =-2 s(1+w), \\
\Delta_{123} & =2 w .
\end{aligned}
$$

The general equations for $\mu_{1}, \mu_{2}$ and the constraints $C$ and $C^{*}$ are the same as in Theorem 1 but with different values of all the $h_{i j}$ functions:

$$
\begin{aligned}
& \mu_{1}=-\frac{h_{43}}{h_{41}} ; \\
& \mu_{2}=\frac{h_{21} h_{43}-h_{23} h_{41}}{h_{41} h_{22}}=\frac{N_{\mu_{2}}}{D_{\mu_{2}}}, \\
& C=h_{31} h_{43}-h_{41} h_{33}=0 .
\end{aligned}
$$


Similar to Theorem 1(a), the constraints $C$ and $C^{*}$ have identical solutions; therefore we use only $C$.

Lemma 7. There exists a region $R f_{\mu_{1}}$ given by (39) in the swplane such that, for any $(s, w) \in R f_{\mu_{1}}, \mu_{1}>0$.

Proof. The method of proof is the same as in Theorem 1. For $\mu_{1}>0, h_{43}$ and $h_{41}$ must have opposite signs. The functions $h_{43}$ and $h_{41}$ are positive in the following regions, respectively:

$$
\begin{aligned}
& R f_{h_{43}+}=\{(s, w) \mid \\
&(-2.73<w<-1 \wedge-0.58(w+1)<s<1) \\
& \vee \\
& \quad(-1<w<0.73 \wedge 0<s<0.58(w+1)) \\
&\vee(w \geq 0.73 \wedge 0<s<1)\}, \\
& R f_{h_{41}+}=\left\{(s, w) \mid w \leq-2 \wedge 0<s<1+\frac{2}{w}\right\} .
\end{aligned}
$$

Therefore the CC region where $\mu_{1}>0$ is determined by

$$
R f_{\mu_{1}}=\left(R f_{h_{43}+} \cap\left(R f_{h_{41}+}\right)^{c}\right) \cup\left(\left(R f_{h_{43}+}\right)^{c} \cap R f_{h_{41}+}\right) .
$$

This completes the proof of Lemma 7.

Lemma 8. There exists a region $R f_{\mu_{2}}$ given by (46) in the swplane such that for any $(s, w) \in R f_{\mu_{2}}, \mu_{2}>0$.

Proof. For the mass ratio $\mu_{2}$ to be positive the functions $N_{\mu_{2}}$ and $D_{\mu_{2}}$ must have the same signs. The denominator of $\mu_{2}$, $D_{\mu_{2}}=h_{41} h_{22}<0$ when $h_{41}$ and $h_{22}$ have opposite sign. The sign analysis of $h_{41}$ is given in (38). The second factor of $D_{\mu_{2}}$, that is, $h_{22}$, is positive in $R f_{h_{22}+}$ and negative in $\left(R f_{h_{22}+}\right)^{c}$ :

$$
\begin{aligned}
& R f_{h_{22}+} \\
& \quad=\left\{(s, w) \mid\left(0<s<1 \wedge-\frac{1}{(s+1)}<w<-\frac{s^{2}}{2}\right)\right\} .
\end{aligned}
$$

Therefore $D_{\mu_{2}}<0$ in

$$
R f_{D_{\mu_{2}}}=\left(R f_{h_{22}+} \cap\left(R f_{h_{41}+}\right)^{c}\right) \cup\left(R f_{h_{41}+} \cap\left(R f_{h_{22}+}\right)^{c}\right)
$$

For the numerator of $\mu_{2}$, that is, $N_{\mu_{2}}=\left(h_{21} h_{43}-h_{23} h_{41}\right)$, to be negative we have the following possibilities with the corresponding regions mentioned alongside them. We will only list the possibilities where we get a nonempty region for $N_{\mu_{2}}<0$ and will ignore all other possibilities: (a) $h_{21}>0, h_{43}<0, h_{23}<0, h_{41}<0$. Consider

$$
\begin{aligned}
& R f_{N_{\mu_{2}} a}=\left\{(s, w) \mid\left(-12<w \leq-1.95 \wedge 0.63+\frac{1}{w}<s\right.\right. \\
& \quad<0.55) \vee\left(-1.95<w \leq-1.59 \wedge 0.63+\frac{1}{w}<s\right. \\
& \quad<-0.58(w+1)) \vee(-1.59<w<-1 \wedge 0<s \\
& \quad<-0.58(w+1)) \vee(-1<w<-0.45 \wedge 0.6>s \\
& \quad>1.2 w+1) \vee(-0.38<w<0 \wedge 0.55>s \\
& \wedge 0.58(w+1))<s\} .
\end{aligned}
$$

(b) $h_{21}>0, h_{43}<0, h_{23}>0, h_{41}<0$. Consider

$$
\begin{gathered}
R f_{N_{\mu_{2}} b}=\{(s, w) \mid s>0.65 \wedge((-5.73<w<-1.95 \wedge s \\
\quad<-0.45(w+1) \wedge s<0.85) \vee(s+0.65 w<1 \\
\wedge(-1<w \leq-0.45 \vee-0.33<w \leq-0.23)))\} .
\end{gathered}
$$

(c) $h_{21}>0, h_{43}<0, h_{23}<0, h_{41}>0$. Consider

$$
R f_{N_{\mu_{2}} c}=\left\{(s, w) \mid\left(w<-2 \wedge 0<s<\frac{2}{w}+1\right)\right\} .
$$

Therefore $N_{\mu_{2}}<0$ in

$$
R f_{N_{\mu_{2}}}=R f_{N_{\mu_{2}} a} \cup R f_{N_{\mu_{2}} b} \cup R f_{N_{\mu_{2}} c} .
$$

Thus the CC region where $\mu_{2}>0$ is given by

$$
R f_{\mu_{2}}=\left(R f_{N_{\mu_{2}}} \cap R f_{D_{\mu_{2}}}\right) \cup\left(\left(R f_{N_{\mu_{2}}}\right)^{c} \cap\left(R f_{D_{\mu_{2}}}\right)^{c}\right) .
$$

This completes the proof of Lemma 8.

The intersection of $R f_{\mu_{1}}$ and $R f_{\mu_{2}}$ will give the region

$$
R f=R f_{\mu_{1}} \cup R f_{\mu_{2}},
$$

which is the central configuration region where both $\mu_{1}$ and $\mu_{2}$ are positive. Region $R f$ will satisfy (3) subject to the constraint $C(s, w)=0$. The intersection of the constraints $C(s, w)=0, C^{*}(s, w)=0$ and the region where all the masses are positive is represented by a line marked $t=1$ in Figure 3 . This confirms the existence of central configuration for the trapezoidal 5 -body problem at $t=1$. There is a continuous family of central configuration when $0<s<0.64$ and $0.2<$ $w<1.8$. There is no central configuration when $0.64<s<1$. This completes the proof of Theorem 1(b).

To complement the analysis given in Theorem 1 a number of graphs are given in Figure 3 for fixed values of $t \in(0,1)$ alongside $t=1$. The general expressions for $\mu_{1}, \mu_{2}$ and the constraints can be derived in the same way as in Theorem 1 .

In each case there is a continuous family of central configurations for both positive and negative values of $w$. In Figures 4(a) and 4(b) 3D regions are given where $\mu_{1}$ and 


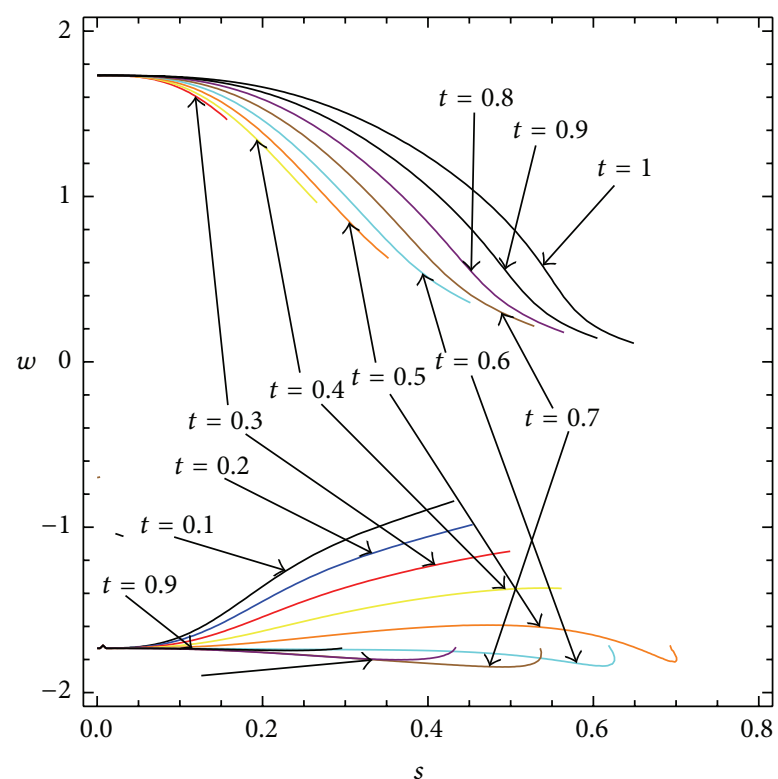

FIGURE 3: Families of central configuration in the case of isosceles trapezoid for values of $t \in(0,1)$. The various lines correspond to values of $t$ as labelled.

$\mu_{2}$ are positive, respectively. Figure 5 gives the intersection of regions given in Figure 4; this will provide the central configuration region subject to the two constraints $C(s, w)=$ $0, C^{*}(s, w)=0$. The part of this region is given in Figure 6 where both the constraints are satisfied which is the central configuration for the trapezoidal 5-body problem where one of the masses is allowed to move symmetrically on the axis of symmetry.

\section{Proof of Theorem 2}

Consider masses $m_{1}, m_{2}$, and $m_{4}$ and masses $m_{2}, m_{3}$, and $m_{5}$ to be collinear. The two sets of collinear masses form a triangle with $m_{2}$ at the intersection of the two sets of collinear masses; see Figure 7. To enforce collinearity take $t=w(1-s)$ in (5). We derive the CC equations from (4) as follows:

$$
\begin{aligned}
& f_{12}=m_{1} h_{31}+m_{4} h_{33}^{*}=0, \\
& f_{24}=m_{1} h_{41}^{*}+m_{4} h_{43}=0, \\
& f_{14}=m_{1} h_{11}+m_{4} h_{13}=0, \\
& f_{15}=m_{1} h_{21}+m_{2} h_{22}+m_{4} h_{23}=0,
\end{aligned}
$$

where

$$
\begin{aligned}
& h_{33}^{*}=\left(R_{15}-R_{24}\right) \Delta_{125}, \\
& h_{41}^{*}=\left(R_{25}-R_{34}\right) \Delta_{243} .
\end{aligned}
$$

We repeat the same procedure as was done in the previous case to obtain the following expressions for the two mass ratios $\mu_{1}=m_{1} / m_{4}, \mu_{2}=m_{2} / m_{4}$ and the geometric constraints $C(s, w)=0$ and $C^{*}(s, w)=0$

$$
\begin{aligned}
\mu_{1} & =\frac{-h_{13}}{h_{11}}, \\
\mu_{2} & =\frac{h_{13} h_{21}-h_{11} h_{23}}{h_{11} h_{22}}, \\
C(s, w) & =h_{13} h_{31}-h_{11} h_{33}^{*}=0, \\
C^{*}(s, w) & =h_{43} h_{11}-h_{13} h_{41}^{*} .
\end{aligned}
$$

Equation (50) determine CC regions in the sw-plane subject to the constraint given by $C$ in (52). First we will analyse $\mu_{1}$ and $\mu_{2}$ and will then discuss $C$ to find CC regions where (4) is satisfied.

Lemma 9. The function $\mu_{1}=-h_{13} / h_{11}$ is positive in

$$
R_{T \mu_{1}}=\left\{(s, w) \mid w>\sqrt{3} \wedge 0<s<f_{1}^{-1}(w)\right\} .
$$

Proof. For the mass ratio $\mu_{1}$ to be positive $h_{13}$ and $h_{11}$ must have opposite signs. Write $h_{13}$ and $h_{11}$ as functions of $s$ and $w:$

$$
\begin{aligned}
& h_{13}(s, w) \\
& =2 s w(s-1)\left(\frac{-1}{8 s^{3}}+\frac{1}{\left((s-1)^{2} w^{2}+(s+1)^{2}\right)^{3 / 2}}\right), \\
& h_{11}(s, w) \\
& =2 w(s-1)\left(\frac{1}{8}-\frac{1}{\left((s-1)^{2} w^{2}+(s+1)^{2}\right)^{3 / 2}}\right) .
\end{aligned}
$$

It is possible to show that $h_{13}$ and $h_{11}$ are positive in the following region:

$$
\begin{aligned}
& R_{h_{13}+}=\{(s, w) \mid w>0 \wedge 0<s<1\}, \\
& R_{h_{11}+}=\{(s, w) \mid(0<w \leq \sqrt{3} \wedge 0<s<1) \\
& \left.\quad \vee\left(w>\sqrt{3} \wedge f_{1}^{-1}(w)<s<1\right)\right\},
\end{aligned}
$$

where

$$
f_{1}(w)=\frac{w^{2}+1}{w^{2}-3} .
$$

Therefore $\mu_{1}$ is positive in

$$
\begin{aligned}
R_{T \mu_{1}} & =\left(R_{h_{13}+} \cap\left(R_{h_{11}+}\right)^{c}\right) \cup\left(\left(R_{h_{13}+}\right)^{c} \cap R_{h_{11}+}\right) \\
& =\left\{(s, w) \mid w>\sqrt{3} \wedge 0<s<f_{1}^{-1}(w)\right\} .
\end{aligned}
$$

Lemma 10. The numerator of $\mu_{2}(s, w), N_{T_{\mu}}(s, w)=h_{13}(s$, $w) h_{21}(s, w)-h_{11}(s, w) h_{23}(s, w)$, is negative in $R_{N_{T \mu_{2}}}(s, w)$ given by (64). 


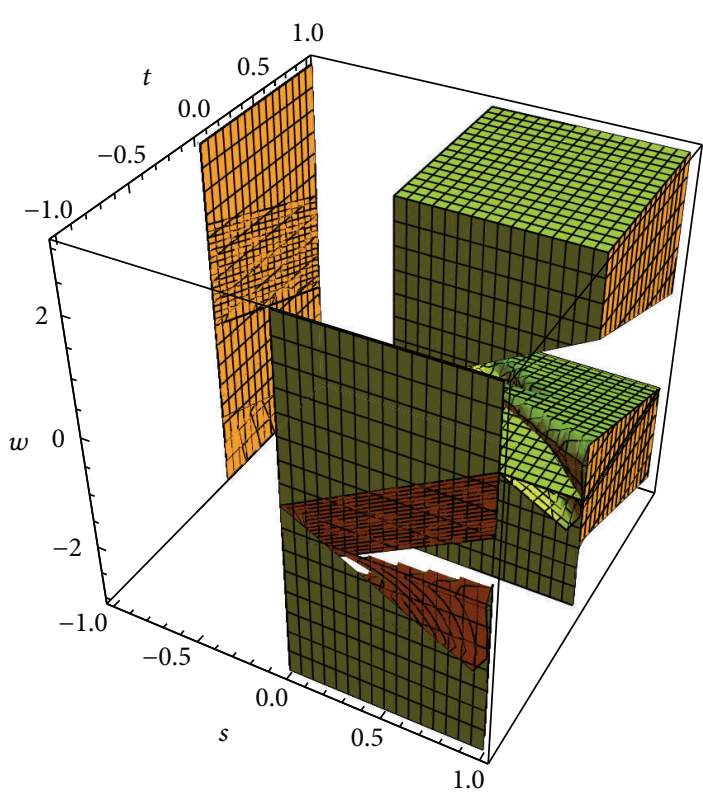

(a)

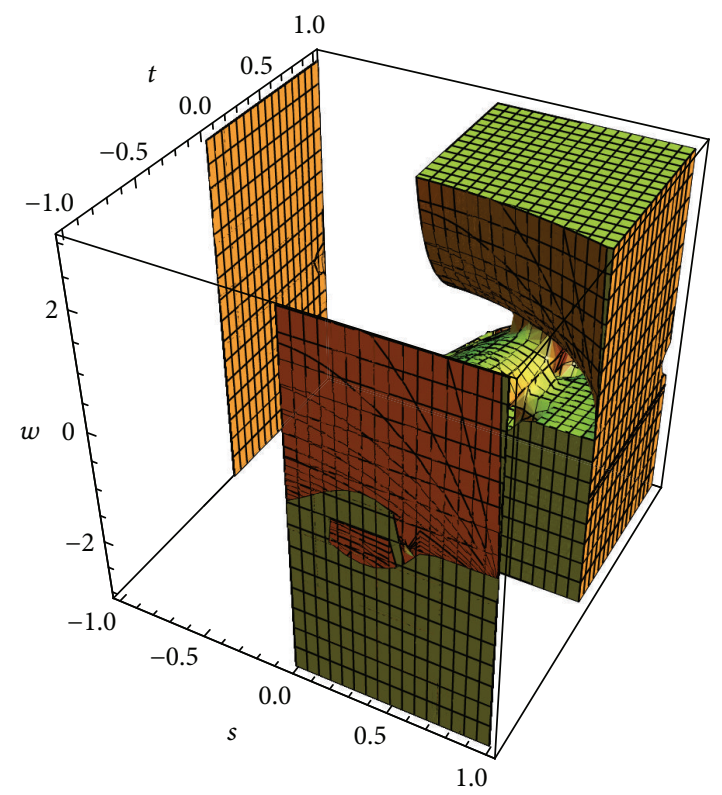

(b)

Figure 4: CC region where (a) $\mu_{1}$ is positive and (b) $\mu_{2}$ is positive subject to the constraints $C(s, w, t)$ and $C^{*}(s, w, t)$.

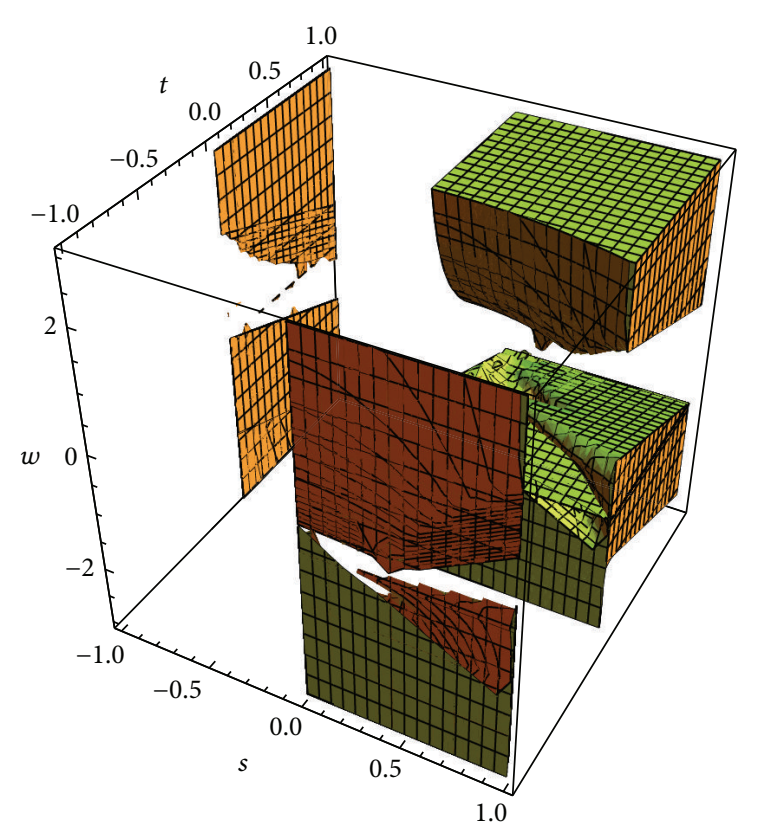

FIGURE 5: CC region where both $\mu_{1}$ and $\mu_{2}$ are positive subject to the constraints $C(s, w, t)$ and $C^{*}(s, w, t)$.

Proof. The function $N_{T \mu_{2}}(s, w)$ involve functions $h_{11}(s, w)$, $h_{13}(s, w), h_{21}(s, w)$, and $h_{23}(s, w)$. The sign analyses of $h_{11}(s, w)$ and $h_{13}(s, w)$ are given in Lemma 9. The remaining functions are given as follows:

$$
h_{21}(s, w)=2 w(s-1)\left(\frac{1}{8}-\frac{1}{(1-s)^{3}\left(1+w^{2}\right)^{3 / 2}}\right)
$$

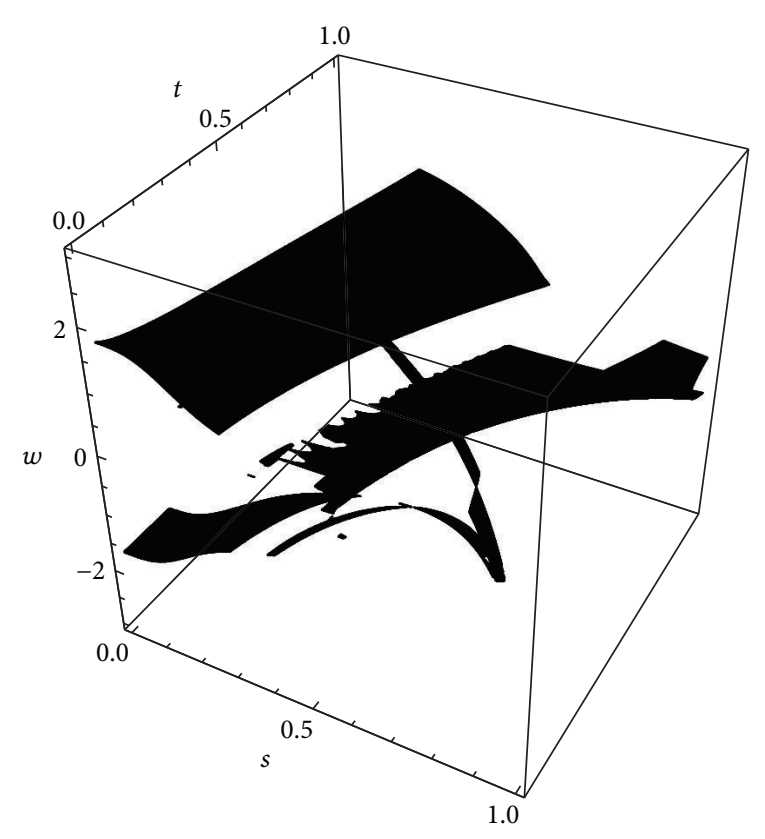

FIGURE 6: CC region in the trapezoidal 5-body problem where all the masses are positive.

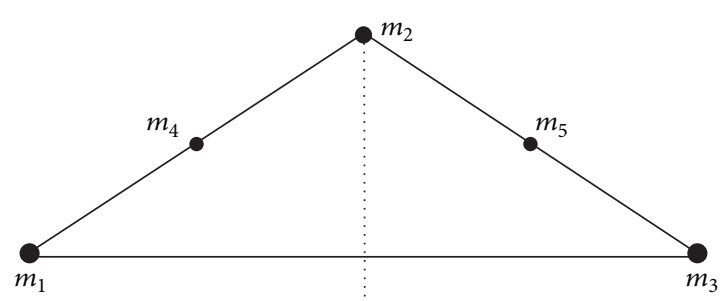

Figure 7: An impossible triangular five-body configuration. 


$$
\begin{aligned}
& h_{23}(s, w) \\
& \quad=2 s w(1-s)\left(\frac{1}{8 s^{3}}-\frac{1}{(s-1)^{3}\left(1+w^{2}\right)^{3 / 2}}\right) .
\end{aligned}
$$

Using the same technique as in Theorem 1 , we find the following regions where $h_{21}$ and $h_{23}$ are positive:

$$
\begin{aligned}
& R_{h_{21}+}=\left\{(s, w) \mid 0<s<1 \wedge 0<w<f_{2}(s)\right\}, \\
& R_{h_{23}+}=\left\{(s, w) \mid \frac{1}{3}<s<1 \wedge 0<w<f_{3}(s)\right\},
\end{aligned}
$$

where

$$
\begin{aligned}
& f_{2}(s)=\sqrt{\frac{-s^{2}+2 s+3}{(s-1)^{2}}}, \\
& f_{3}(s)=\sqrt{\frac{3 s^{2}+2 s-1}{(s-1)^{2}}} .
\end{aligned}
$$

For the numerator of $\mu_{2}$, that is, $N_{T \mu_{2}}=\left(h_{13} h_{21}-h_{11} h_{23}\right)$, to be negative we have the following possibilities with the corresponding regions mentioned alongside them. As before we list only the possibilities where $N_{T \mu_{2}}<0$ return nonempty regions:

(a) $h_{13}>0, h_{21}<0, h_{11}<0, h_{23}<0 ; R_{h_{13}+} \cap\left(R_{h_{21}+}\right)^{c} \cap$ $\left(R_{h_{11}+}\right)^{c} \cap\left(R_{h_{23}+}\right)^{c}$. Consider

$$
\begin{aligned}
& R_{N_{T \mu_{2}}} a \\
& \quad=\left\{(s, w) \mid w>\sqrt{3} \wedge 0<s<1-2 \sqrt{\frac{1}{w^{2}+1}}\right\} .
\end{aligned}
$$

(b) $h_{13}>0, h_{21}>0, h_{11}<0, h_{23}<0 ; R_{h_{13}+} \cap R_{h_{21}+} \cap$ $\left(R_{h_{11}}\right)^{c} \cap\left(R_{h_{23}}\right)^{c}$. Consider

$$
\begin{gathered}
R_{N_{T_{\mu_{2}}}} b^{*}=\{(s, w) \mid \sqrt{3}<w \leq 3.078 \wedge 1 \\
-2\left(w^{2}+1\right)^{-1 / 2}<s<f_{1}^{-1} \vee w>3.078 \wedge 1 \\
\left.-2 \sqrt{\frac{1}{w^{2}+1}}<s<f_{1}(w)-2 \sqrt{\frac{f_{1}(w)}{w^{2}-3}}\right\} .
\end{gathered}
$$

$N_{T \mu_{2}}<0$ in the following part of $R N_{T \mu_{2}} b^{*}$

$$
\begin{aligned}
& R_{N_{T_{\mu_{2}}}} b=\left\{(s, w) \mid w>1.9 \wedge 1-2\left(w^{2}+1\right)^{-1 / 2}<s\right. \\
& \left.<\left(w^{2}-1.3\right)\left(w^{2}+10\right)^{-1}\right\} .
\end{aligned}
$$

Therefore $N_{T \mu_{2}}<0$ in

$$
\begin{aligned}
& R_{N_{T \mu_{2}}}=R_{N_{T \mu_{2}}} a \cup R_{N_{T_{\mu_{2}}}} b=\{(s, w) \mid \\
& \left(w>1.9 \wedge 1-2 \sqrt{\frac{1}{w^{2}+1}}<s<\frac{w^{2}-1.3}{w^{2}+10}\right) \\
& \left.\vee\left(w>\sqrt{3} \wedge 0<s<1-2 \sqrt{\frac{1}{w^{2}+1}}\right)\right\} .
\end{aligned}
$$

This completes the proof of Lemma 10.

Lemma 11. (a) The function $D_{T \mu_{2}}(s, w)=h_{11}(s, w) h_{22}(s, w)$ which is the denominator of $\mu_{2}$ is positive in $R_{D_{T_{\mu_{2}}}}$ given by (70).

(b) The central configuration region where $\mu_{2}>0$ is given by $R_{T_{\mu 2}}$.

Proof. The function $h_{22}$ is given below as function of $s$ and $w$ :

$$
\begin{aligned}
& h_{22}(s, w) \\
& \quad=2 w\left(\frac{1}{\left(s^{2}+(2-s)^{2} w^{2}\right)^{3 / 2}}-\frac{1}{\left(w^{2}+1\right)^{3 / 2}}\right) .
\end{aligned}
$$

Using the same technique as in Theorem 1, we find that $h_{22}(s, w)$ is positive in the following region:

$$
\begin{gathered}
R_{h_{22}+}=\left\{(s, w) \mid\left(0<w \leq \frac{1}{\sqrt{3}} \wedge 0<s<1\right)\right. \\
\left.\vee\left(\frac{1}{\sqrt{3}}<w<1 \wedge \frac{3 w^{2}-1}{w^{2}+1}<s<1\right)\right\} .
\end{gathered}
$$

For $h_{11} h_{22}=D_{T \mu_{2}}>0 ; h_{11}$ and $h_{22}$ must have the same signs. Both $h_{11}$ and $h_{22}$ are positive in $R_{h_{11}+}$ and $R_{h_{22}+}$, respectively. Therefore, $D_{T \mu_{2}}>0$ in $R_{h_{11}+} \cap R_{h_{22}+}$ and $\left(R_{h_{11}+}\right)^{c} \cap$ $\left(R_{h_{22}+}\right)^{c}$ :

$$
\begin{aligned}
& \left(R_{h_{11^{+}}}\right)^{c} \cap\left(R_{h_{22}+}\right)^{c} \\
& \quad=\left\{(s, w) \mid w>\sqrt{3} \wedge 0<s<f_{1}^{-1}(w)\right\} .
\end{aligned}
$$

Similarly $R_{h_{11}+} \cap R_{h_{22}+}$ can be found as follows:

$$
\begin{gathered}
R_{h_{11}+} \cap R_{h_{22}+}=\left\{(s, w) \mid\left(0<w \leq \frac{1}{\sqrt{3}} \wedge 0<s<1\right)\right. \\
\left.\vee\left(\frac{1}{\sqrt{3}}<w<1 \wedge \frac{3 w^{2}-1}{w^{2}+1}<s<1\right)\right\} .
\end{gathered}
$$




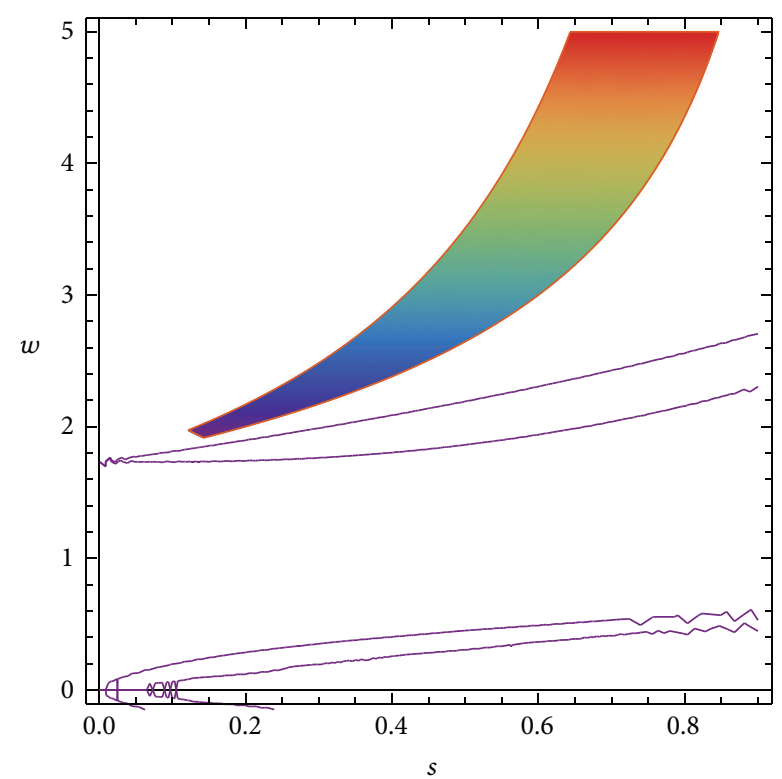

FIGURE 8: Region $R_{T}$ is given by the colored portion of the graph and the constraints $C(s, w)=0, C^{*}(s, w)=0$ are given by the purple lines. There is no intersection between the CC region and the constraints.

Therefore $D_{T \mu_{2}}>0$ in $\left(R_{h_{11}+} \cap R_{h_{22}+}\right) \cup\left(\left(R_{h_{11}}\right)^{c} \cap\left(R_{h_{22}+}\right)^{c}\right)=$ $R D_{T \mu_{2}}$, where

$$
\begin{aligned}
R D_{T \mu_{2}} & =\left\{(s, w) \mid\left(w<0 \wedge s<f_{1}^{-1}(w)\right) \vee(0<w\right. \\
< & \left.1 \wedge \frac{3 w^{2}-1}{w^{2}+1}<s<1\right) \vee(1<w \leq \sqrt{3} \wedge 1<s \\
< & \left.\frac{3 w^{2}-1}{w^{2}+1}\right) \vee(w>\sqrt{3} \\
\wedge & \left.\left.\left(0<s<f_{1}^{-1}(w) \vee 1<s<\frac{3 w^{2}-1}{w^{2}+1}\right)\right)\right\} .
\end{aligned}
$$

This completes the proof of Lemma 11(a).

Thus the CC region $\left(R_{T \mu_{2}}\right)$ where $\mu_{2}>0$ is determined from Lemmas 10 and 11(a) as follows:

$$
R_{T \mu_{2}}=\left(\left(R_{N_{T \mu_{2}}}\right)^{c} \cap R_{D_{T \mu_{2}}}\right) \cup\left(R_{N_{T \mu_{2}}} \cap\left(R_{D_{T \mu_{2}}}\right)^{c}\right) .
$$

From Lemmas 9 and 11(b), the region where both $\mu_{1}$ and $\mu_{2}$ are positive is found by taking the intersection of $R_{T \mu_{1}}$ and $R_{T \mu_{2}}$ and is given by

$$
R_{T}=R_{T \mu_{1}} \cap R_{T \mu_{2}} .
$$

It is clear from Figure 8 that the constraint $C(s, w)=0$ does not intersect the region where all the masses are positive. Hence no central configurations of this particular type are possible.

\section{Conclusions}

We studied the central configuration of different types of symmetric 5-body problems. All the problems investigated have two pairs of equal masses. In one case two pairs of equal masses are placed at the vertices of an isosceles trapezoid and the fifth body not necessarily equal to the other masses was allowed to take various positions on the axis of symmetry. Initially we formed expressions for mass ratios and derived regions of central configuration for positive masses. The existence of continuous family of central configurations is shown to exist for trapezoids of various sizes. It is also shown that it is possible for the fifth mass to be both inside the trapezoid and outside the trapezoid. In the second case the five masses are arranged in two sets of three collinear masses such that one of the masses is at the intersection of the two collinear sets, hence making a triangle; similarly expressions are formed for mass ratios to derive regions of central configuration for positive masses. It was shown that no such type of central configuration is possible.

\section{Conflict of Interests}

The authors declare that there is no conflict of interests regarding the publication of this paper.

\section{Acknowledgment}

The authors thank the deanship of research at the University of Ha'il, Saudi Arabia, for funding this work under Grant no. SM14007.

\section{References}

[1] M. Gidea and J. Llibre, "Symmetric planar central configurations of five bodies: euler plus two," Celestial Mechanics and Dynamical Astronomy, vol. 106, no. 1, pp. 89-107, 2010.

[2] C. Deng and S. Zhang, "Planar symmetric concave central configurations in Newtonian four-body problems," Journal of Geometry and Physics, vol. 83, pp. 43-52, 2014.

[3] W. D. MacMillan and W. Bartky, "Permanent configurations in the problem of four bodies," Transactions of the American Mathematical Society, vol. 34, no. 4, pp. 838-875, 1932.

[4] J. Llibre and L. F. Mello, "New central configurations for the planar 5-body problem," Celestial Mechanics \& Dynamical Astronomy, vol. 100, no. 2, pp. 141-149, 2008.

[5] C. Simo, "Relative equilibrium solutions in the four-body problem," Celestial Mechanics, vol. 18, no. 2, pp. 165-184, 1978.

[6] T.-L. Lee and M. Santoprete, "Central configurations of the five-body problem with equal masses," Celestial Mechanics and Dynamical Astronomy, vol. 104, no. 4, pp. 369-381, 2009.

[7] M. Shoaib, A. Sivasankaran, and A. Kashif, "Central configurations in the collinear 5-body problem," Turkish Journal of Mathematics, vol. 38, no. 3, pp. 576-585, 2014.

[8] X. Su and C. Deng, "Two classes of stacked central configurations for the spatial $2 \mathrm{n}+1$-body problem: nested regular polyhedra plus one," Journal of Geometry and Physics, vol. 76, pp. 1-9, 2014. 
[9] T. Ouyang and Z. Xie, "Collinear central configuration in fourbody problem," Celestial Mechanics and Dynamical Astronomy, vol. 93, no. 1, pp. 147-166, 2005.

[10] S. Smale, "Mathematical problems for the next century," The Mathematical Intelligencer, vol. 20, no. 2, pp. 7-15, 1998.

[11] M. Hampton and R. Moeckel, "Finiteness of relative equilibria of the four-body problem," Inventiones Mathematicae, vol. 163, no. 2, pp. 289-312, 2006.

[12] A. Albouy and V. Kaloshin, "Finiteness of central configurations of five bodies in the plane," Annals of Mathematics, vol. 176, no. 1, pp. 535-588, 2012.

[13] M. Hampton and A. Jensen, "Finiteness of spatial central configurations in the five-body problem," Celestial Mechanics and Dynamical Astronomy, vol. 109, no. 4, pp. 321-332, 2011.

[14] A. Albouy and R. Moeckel, "The inverse problem for collinear central configurations," Celestial Mechanics and Dynamical Astronomy, vol. 77, no. 2, pp. 77-91, 2000.

[15] Z. Xie, "Inverse problem of central configurations and singular curve in the collinear 4-body problem," Celestial Mechanics and Dynamical Astronomy, vol. 107, no. 3, pp. 353-376, 2010.

[16] M. Hampton, "Stacked central configurations: new examples in the planar five-body problem," Nonlinearity, vol. 18, no. 5, pp. 2299-2304, 2005.

[17] M. Shoaib, I. Faye, and A. Sivasankaran, "Some special solutions of the rhomboidal five-body problem," in Proceedings of the International Conference on Fundamental and Applied Sciences (ICFAS '12), vol. 1482 of AIP Conference Proceedings, pp. 496501, AIP Publishing, Kuala Lumpur, Malaysia, June 2012.

[18] Z. Xie, "Isosceles trapezoid central configurations of the Newtonian four-body problem," Proceedings of the Royal Society of Edinburgh: Section A Mathematics, vol. 142, no. 3, pp. 665-672, 2012.

[19] M. Shoaib, "Regions of central configurations in a symmetric 4 + 1-body problem," Advances in Astronomy, vol. 2015, Article ID 284189, 1 page, 2015. 

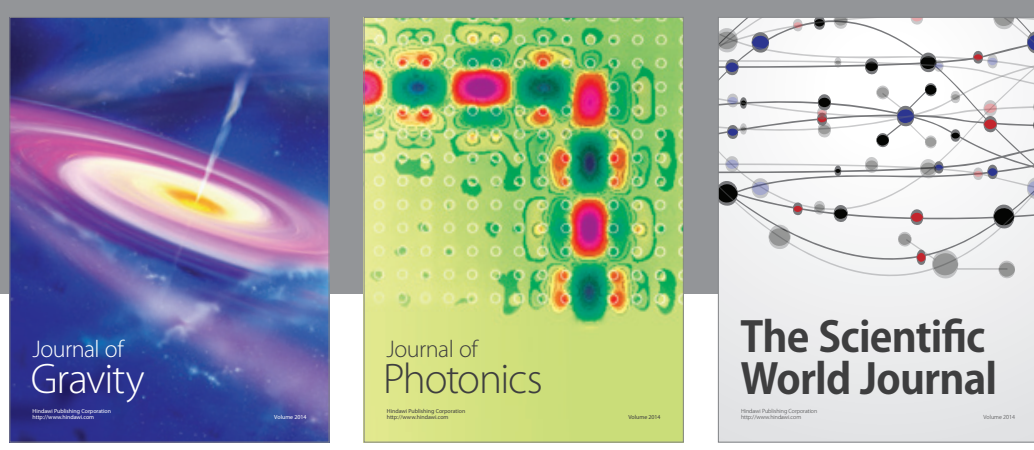

The Scientific World Journal
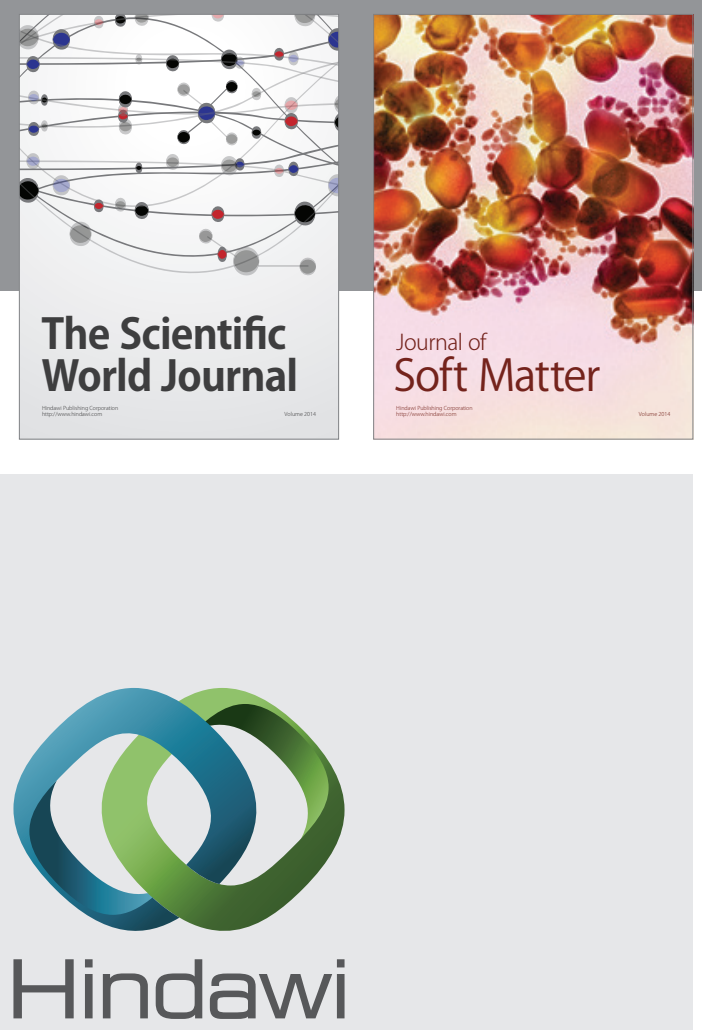

Submit your manuscripts at

http://www.hindawi.com

nternational Journal of

Statistical Mechanics
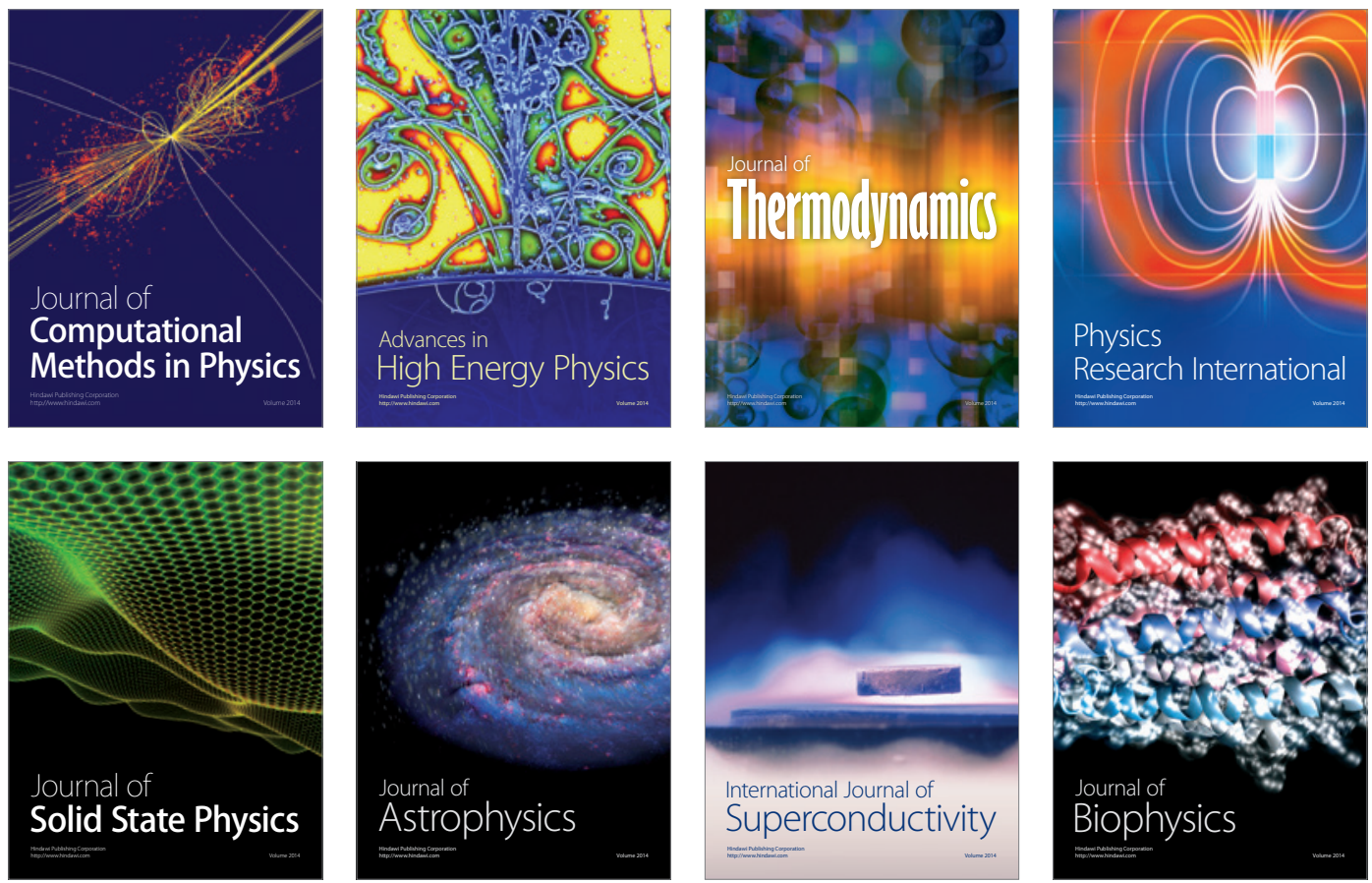
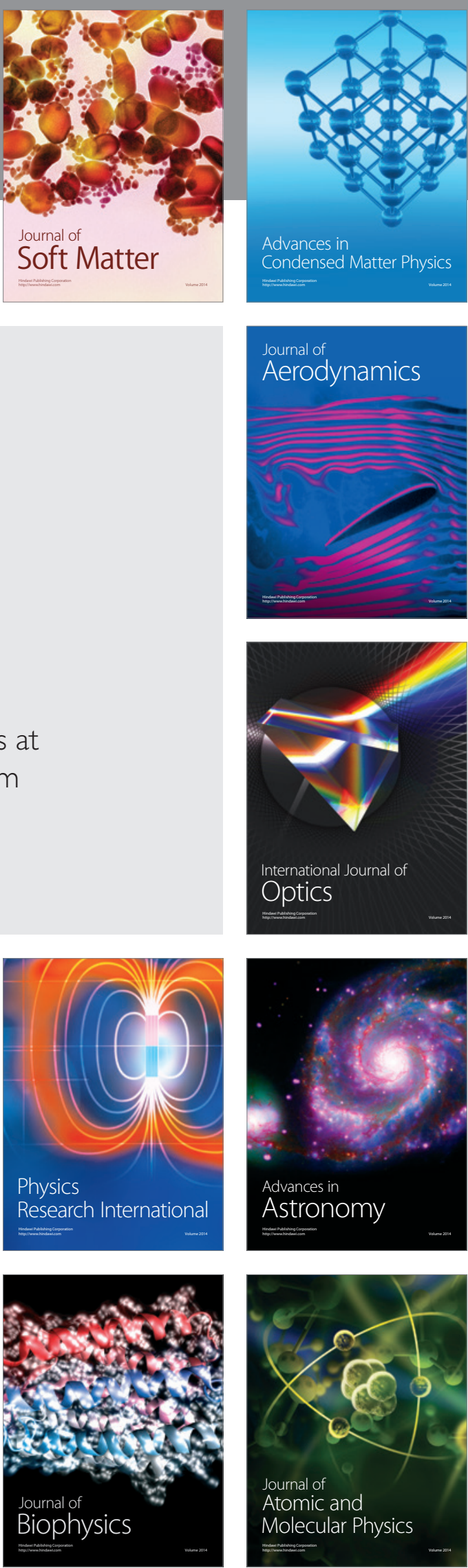